\title{
Desnutrição, inflamação e outros fatores de risco para doença cardiovascular em pacientes sob diálise peritoneal
}

\author{
Malnutrition, inflammation and other risk factors \\ for cardiovascular diseases in patients \\ undergoing peritoneal dialysis
}

\author{
Maria Helena Lima GUSMÃO' \\ Alessandra Fortes ALMEIDA² \\ Lia Santa Isabel MICHELLE ${ }^{2}$ \\ Mayara Nogueira MOREIRA² \\ Sara Ribeiro CAMPOS² \\ Luis José Cardoso PEREIRA ${ }^{3}$ \\ Jairza Maria Barreto MEDEIROS ${ }^{1}$
}

\section{RE S U M O}

\section{Objetivo}

Identificar a ocorrência de desnutrição, inflamação e outros fatores de risco para doença cardiovascular em pacientes sob diálise peritoneal.

\section{Métodos}

Estudo transversal, envolvendo 61 pacientes em duas unidades de diálise em Salvador (BA). O estado nutricional foi avaliado por meio da Avaliação Subjetiva Global. Realizou-se a dosagem de proteína C reativa ultrassensível para determinar inflamação. Foram estudados fatores de risco tradicionais para doença cardiovascular, como gênero, idade, etilismo, tabagismo, sedentarismo, hipertensão, diabetes, dislipidemia e hábitos alimentares e fatores não tradicionais, como alterações no metabolismo de cálcio, fósforo e anemia.

\section{Resultados}

Nessa população, 36,0\% dos pacientes encontravam-se desnutridos e 49,2\% com processo inflamatório. Desnutrição e inflamação foram evidenciadas em $21,3 \%$ dos pacientes. O grupo de pacientes desnutridos

\footnotetext{
1 Universidade Federal da Bahia, Escola de Nutrição, Programa de Pós-Graduação em Alimentos, Nutrição e Saúde. R. Araújo Pinho, 32, Canela, 40110-150, Salvador, BA, Brasil. Correspondência para/Correspondence to: M.H.L. GUSMÃO. E-mail: <mhgusmao@terra.com.br>.

${ }^{2}$ Universidade Federal da Bahia, Escola de Nutrição. Salvador, BA, Brasil.

${ }^{3}$ Universidade Federal da Bahia, Complexo Hospitalar Universitário Professor Edgard Santos. Salvador, BA, Brasil.
} 
336 | M.H.L. GUSMÃO et al.

apresentou níveis maiores de proteína $C$ reativa ultrassensível do que o de pacientes com bom estado nutricional $(p=0,04)$. A elevada ocorrência dos outros fatores risco para doença cardiovascular também foi observada na população.

\section{Conclusão}

A desnutrição ainda é frequente nos pacientes sob diálise peritoneal, assim como a inflamação. Pacientes desnutridos apresentam maiores níveis séricos de proteína $C$ reativa ultrassensível do que aqueles com bom estado nutricional. Observa-se que muitos dos fatores de risco evidenciados na população estudada são passíveis de terapêutica.

Termos de indexação: Desnutrição. Diálise Peritoneal. Doença Cardiovascular. Inflamação.

\section{A B S T R A C T}

\section{Objective}

This study aimed to identify the occurrence of malnutrition, inflammation and other risk factors for cardiovascular diseases in patients undergoing peritoneal dialysis.

\section{Methods}

This is a cross-sectional study comprising 61 patients from two dialysis units in Salvador (Bahia, Brazil). Nutritional status was determined by the global subjective assessment and inflammation by the ultra sensitive c-reactive protein levels. Traditional risk factors for cardiovascular diseases, such as gender, age, consumption of alcoholic beverages, smoking, inactivity, hypertension, diabetes, dyslipidemia and food habits, as well as non-traditional factors such as disturbed calcium and phosphorus metabolism and anemia, were investigated.

\section{Results}

Malnutrition was found in $36.0 \%$ of the patients in the population under study whereas inflammation was present in $49.2 \%$ of them. Patients with both malnutrition and inflammation were shown to comprise $21.3 \%$ of the sample. Ultra sensitive c-reactive protein levels were found to be higher $(p=0.04)$ in malnourished patients than in those with good nutritional status. High rates of other risk factors for cardiovascular disease were also observed in this population.

\section{Conclusion}

Malnutrition and inflammation are still common among patients undergoing peritoneal dialysis. Ultra sensitive c-reactive protein levels were found to be higher among malnourished patients than in well-nourished patients. Many of the risk factors found in the studied population are treatable.

Indexing terms: Malnutrition. Peritoneal Dialysis. Cardiovascular Disease. Inflammation.

\section{N T R O D U ÇÃ O}

Apesar dos avanços no tratamento dialítico, a mortalidade na população com Doença Renal Crônica (DRC) continua elevada, sendo influenciada por diversos fatores, dentre eles a desnutrição ${ }^{1}$. A Doença Cardiovascular (DCV), contudo, é a principal causa de óbito em pacientes com $D R C$, principalmente naqueles em diálise ${ }^{2}$. A taxa de mortalidade por DCV em pacientes em diálise é aproximadamente de $9 \%{ }^{3}$.

Fatores de risco para DCV considerados tradicionais, como idade avançada, gênero masculino, hipertensão arterial, dislipidemia, diabetes mellitus, tabagismo, etilismo e inatividade física, são responsáveis por grande parte da alta mortalidade observada nessa população ${ }^{3-8}$. Pacientes em Diálise Peritoneal (DP), além de apresentarem alta prevalência de hipertensão e diabetes, também podem desenvolver hipertrigliceridemia grave, provavelmente devido à absorção de glicose do dialisato. Alterações nos níveis de colesterol, devido à perda de fração de colesterol de Lipoproteína de Alta Densidade (HDL-c), apolipoproteína A e lecitina colesterol acil trasnferase para o líquido de diálise também têm sido observadas².

Fatores de risco não tradicionais para DCV também estão comumente presentes nessa popu- 
lação, como hiper-homocisteinemia, estresse oxidativo, alteração do metabolismo do cálcio e do fósforo, anemia e principalmente o binômio inflamação e desnutrição ${ }^{3,4,7,8}$. A prevalência de inflamação é descrita em torno de $61,9 \%$ na população em DP, mesmo em condições clínicas estáveis ${ }^{9}$. A prevalência de desnutrição varia de 32,9\% a $51,2 \%$ na forma leve a moderada e de $2,6 \%$ a 4,2\% para desnutrição grave ${ }^{1,10-12}$. A associação entre inflamação, desnutrição e aterosclerose tem sido relacionada à alta mortalidade por $\mathrm{DCV}$ em pacientes portadores de $\mathrm{DRC}^{3,7}$.

Considerando, assim, a importância da desnutrição no prognóstico dos pacientes em DP e a participação do binômio inflamação e desnutrição no desenvolvimento da DCV, este estudo teve como objetivo identificar a ocorrência de desnutrição, inflamação e outros fatores de risco para DCV em pacientes sob DP.

\section{MÉ T O D O S}

O estudo observacional transversal foi realizado nas unidades de tratamento dialítico do Complexo Hospitalar Universitário Professor Edgard Santos (HUPES) e da Clínica São Marcos (CSM), Salvador (BA), no período de março a agosto de 2007, e envolveu 61 pacientes. Esse número representa 33\% da população em diálise peritonial de Salvador. Participaram do estudo pacientes adultos ( $\geq 20$ e $<60$ anos) e idosos ( $\geq 60$ anos) de ambos os gêneros, em Diálise Peritoneal Ambulatorial Contínua (DPAC) ou Diálise Peritoneal Automatizada (DPA) há pelo menos três meses, em uso de dieta via oral e com clearance de creatinina menor do que $10 \mathrm{~mL} / \mathrm{min} / 1,73 \mathrm{~m}^{2}$ para pacientes não diabéticos e menor do que $15 \mathrm{~mL} / \mathrm{min}$ $1,73 \mathrm{~m}^{2}$ para pacientes diabéticos.

Foram considerados critérios de exclusão: ser portador de câncer, síndrome da imunodeficiência adquirida e/ou doença hepática, possuir história clínica de doença inflamatória crônica, peritonite, abandono da terapia dialítica nos últimos três meses, história de alcoolismo, insucesso no transplante renal nos últimos seis meses, uso de corticosteroides, uso de nutrição enteral e/ou nutrição parenteral exclusiva ou associada à dieta via oral e DCV. Como DCV foram consideradas: doença vascular periférica, hipertrofia do ventrículo esquerdo, insuficiência cardíaca, insuficiência coronariana, infarto do miocárdio, angina e acidente vascular encefálico.

Para caracterizar a população, foram utilizados dados demográficos, como idade e gênero, e dados clínicos, como a etiologia da doença renal, o tipo de DP e o tempo de DP em meses. O estado nutricional dos pacientes foi avaliado por meio da Avaliação Subjetiva Global (ASG) e classificado em desnutrição grave, desnutrição leve ou moderada e estado nutricional adequado ${ }^{1,13}$. Para avaliar a presença de inflamação, foi utilizada a dosagem de Proteína C Reativa Ultrasensível (PCR US) por meio do método de Nefelometria ${ }^{3}$. Foram considerados com processo inflamatório e em risco de doença cardiovascular os indivíduos com valores acima de $3 \mathrm{mg} / \mathrm{L}^{14}$.

Foram adotados como fatores de risco demográficos para DCV, gênero masculino e idade maior do que 60 anos. Informações sobre fatores clínicos, como hipertensão arterial sistêmica e diabetes mellitus, foram coletadas do prontuário médico. A presença de dislipidemia foi avaliada por meio das dosagens de Colesterol Total (CT), HDLc, fração de colesterol de Lipoproteína de Baixa Densidade (LDL-C), Lipoproteína de Muito Baixa Densidade (VLDL) e Triglicérides (TG), seguindo as recomendações determinadas pela Sociedade Brasileira de Cardiologia (SBC) ${ }^{14}$. Os níveis séricos de CT e TG foram determinados pelo método colorimétrico enzimático automatizado, o nível de HDLc, por meio do método direto e os níveis de LDLc e VLDLc, pela Fórmula de Friedewald ${ }^{15}$.

Como fatores de risco comportamentais foram considerados ingestão de bebida alcoóli$\mathrm{ca}^{16}$, quantidade de etanol consumida por dia ${ }^{17}$, hábito de fumar ${ }^{16}$ e sedentarismo ${ }^{18}$. Classificou-se como consumo alto de etanol para homens valores $>30 \mathrm{~mL} /$ dia e para mulheres $>15 \mathrm{~mL} / \mathrm{dia}^{17}$. O hábito de fumar foi classificado em duas cate- 
gorias: consumo $<20$ cigarros/dia e consumo $\geq 20$ cigarros/dia ${ }^{16}$.

Dentre os fatores de riscos alimentares foram avaliados o Valor Calórico Total (VCT) da dieta, a ingestão de carboidratos, lipídeos totais, gordura saturada, gordura poli-insaturada, gordura monoinsaturada, colesterol e fibras ${ }^{14}$. A avaliação da ingestão alimentar habitual foi realizada utilizando o registro alimentar de três dias (dois dias durante a semana e um do final de semana). Para o cálculo da ingestão de nutrientes foi utilizado o programa Diet Pro versão 4.0. ${ }^{19}$, tomando-se como referência a Tabela Americana de Composição dos Alimentos ${ }^{19}$. Alimentos regionais ausentes na tabela foram acrescidos ao programa, utilizando-se a Tabela de Composição Química dos Alimentos do Estudo Nacional da Despesa Familiar ${ }^{20}$ ou a Tabela de Composição Química dos Alimentos de Philipp ${ }^{21}$. A glicose absorvida do dialisato também foi acrescida ao consumo alimentar e foi estimada pela equação proposta por Grodstein et al.22. O Consumo total de nutrientes foi comparado às recomendações específicas da Sociedade Brasileira de Cardiologia ${ }^{14}$. Já os valores de absorção de glicose foram comparados às estimativas de Tiogo et al. ${ }^{23}$. Como parâmetro de referência para o consumo alimentar de carboidrato, foi utilizada a recomendação específica para pacientes renais em $D^{23}$.

Como fatores de risco clínicos não tradicionais para DCV foram avaliados os níveis de Paratormônio Intacto (PTHi), cálcio e fósforo sérico e produto cálcio $x$ fósforo, pois alterações nesses parâmetros podem predispor a calcificação vascular. Para análise do paratormônio intacto, foi usado o método da quimioluminescência, e para as dosagens de cálcio e fósforo, o método titulométrico e de Basques e Lustosa respectivamente. Os valores de cálcio foram corrigidos pelos níveis de albumina sérica ${ }^{24}$. A dosagem de albumina foi realizada pelo método verde de bromocresol. Os valores encontrados foram comparados aos referenciados pelo National Kidney Formulation $\mathrm{K} / \mathrm{DOQ}{ }^{24}$. Além disso, a presença de anemia foi verificada por meio da dosagem de hemoglobina sérica ${ }^{25}$. Os resultados foram comparados aos preconizados pelo National Kidney Formulation ${ }^{25}$.

Os dados foram apresentados como Média (M), Desvio-Padrão (DP) ou mediana (valores mínimo e máximo) para variáveis contínuas e percentagem para variáveis categóricas. Para análise estatística, os dados obtidos foram classificados pelo teste de Kolmogorov-Smirnov como de distribuição normal (dados paramétricos) e não normal (dados não-paramétricos). Na comparação entre dois grupos, foi utilizado o teste $t$ de Student para os dados paramétricos e de Mann-Whitney para os não-paramétricos. Para comparação entre mais de dois grupos, foi empregada análise de variância ANOVA seguida do teste Tukey para os dados paramétricos e, para os dados não-paramétricos, o teste de Kruskal-Wallis seguido do teste de Mann-Whitney. As proporções foram comparadas pelo teste do qui-quadrado ou teste exato de Fisher. O Programa Statistical Package for Social Science (SPSS) ${ }^{\circledR}$ (Versão 13.0) foi utilizado para todas as análises estatísticas, com significância de $5 \%(p<0,05)$.

O protocolo deste estudo foi aprovado pelo Comitê de Ética em Pesquisa da Escola de Nutrição da Universidade Federal da Bahia, parecer $n^{\circ} 04 / 2006$.

\section{RESULTADOS}

A população estudada apresentou média de idade de 51,97 e DP=16,45 anos. Verificou-se predominância de pacientes adultos (63,9\%) e do gênero feminino (59,0\%). Observou-se que $41,0 \%$ dos pacientes tiveram como causa de doença renal a nefroesclerose hipertensiva; $23,0 \%$, as glomerulopatias; $19,7 \%$, a nefropatia diabética, e 16,4\%, outras causas. Com relação ao tipo de doença peritoneal, evidenciou-se que $86,9 \%$ dos pacientes realizavam DPAC, enquanto 13,1\% DPA. A mediana do tempo em DP foi de 13 meses (3-94).

A avaliação do estado nutricional por meio da ASG permitiu verificar que somente $1,6 \%$ dos 
pacientes apresentavam desnutrição grave, enquanto 34,4\% apresentavam desnutrição na forma leve a moderada. Da população estudada, 49,2\% apresentaram níveis de PCR US acima de $3 \mathrm{mg} / \mathrm{L}$ e $21,3 \%$ se encontravam desnutridos e com processo inflamatório. Nessa população, o nível sérico de PCR US, expresso em mediana, foi $2,5 \mathrm{mg} / \mathrm{L}(0,08-28,40)$. Quando se dividiu a população em dois grupos - bom estado nutricional e desnutrido -, foi evidenciado que o grupo desnutrido apresentou níveis maiores de PCR US - expresso em mediana, $6,91 \mathrm{mg} / \mathrm{dL}(0,23-28,40)$ - em relação aos apresentados pelo grupo com bom estado nutricional, $2,13 \mathrm{mg} / \mathrm{dL}(0,08-16,50),(p=0,04)$ (Figura 1). O nível sérico de albumina nessa população foi em média $M=3,06, D P=0,52 \mathrm{mg} / \mathrm{dL}$, de forma que $75,4 \%$ dos pacientes apresentavam níveis de albumina sérica menor do que 3,5mg/dL.

Em relação aos fatores de risco tradicionais demográficos e comportamentais, observou-se que $36,1 \%$ dos indivíduos estudados eram idosos e $41,0 \%$ do gênero masculino. A frequência de etilismo foi de $16,4 \%$, e dentre os que consumiam bebida alcoólica, $60 \%$ apresentavam ingestão elevada de etanol. Além disso, 44,3\% dos pacientes foram classificados como ex-etilistas. A frequência de tabagismo foi de $3,3 \%$. Apenas um paciente apresentou consumo de mais de 20 cigarros por dia. A frequência de ex-tabagistas, contudo, foi de $34,4 \%$. O sedentarismo foi evidenciado em $86,9 \%$ dos estudados.

Dentre os fatores clínicos, observou-se que a frequência de hipertensão arterial sistêmica nessa população foi de $77,0 \%$ e a de diabetes $24,6 \%$ e que $91,8 \%$ dos pacientes apresentavam dislipidemia. A elevação do CT foi evidenciada em $50,8 \%$ da população, hipertrigliceridemia em $62,3 \%$, níveis elevados de LDL-c em $31,1 \%$ e níveis baixos de HDL-c em $73,8 \%$.

Quanto ao perfil de consumo alimentar, observou-se que a população estudada apresentou ingestão energetica diária média de $M=1770,14, D P=573,11 \mathrm{kcal}$. De acordo com a distribuição percentual dos macronutrientes, iden-

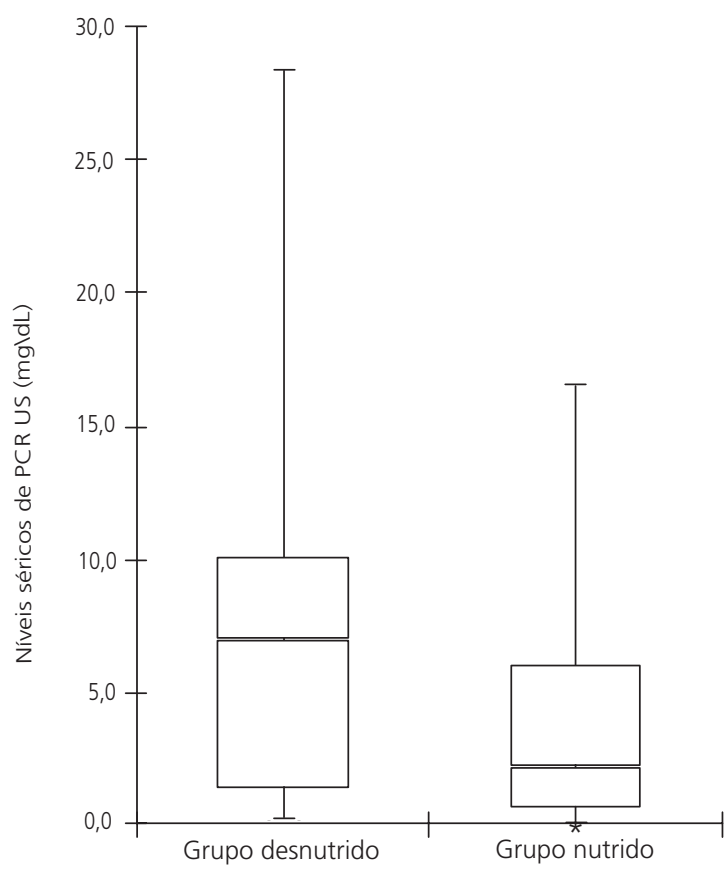

Figura 1. Nível sérico de PCR US de pacientes sob diálise peritoneal contínua, expresso em mediana no grupo desnutrido e bom estado nutricional, por meio da ASG. Salvador (BA), 2007.

Nota: Teste de Mann-Whitney, $p=0,04$; PCR US: proteína $C$ reativa ultrasensssivel.

tificou-se que $M=64,08, D P=8,27 \%$ correspondem aos carboidratos, dos quais $M=47,22$, $D P=9,26 \%$ são oriundos da dieta e $M=16,82$, $\mathrm{DP}=8,99 \%$ da glicose absorvida do dialisato. $\mathrm{O}$ consumo de lipídeo representou $M=22,49$, $\mathrm{DP}=6,87 \%$ do $\mathrm{VCT}$, e as gorduras saturadas, poli-insaturadas e monoinstauradas representaram, respectivamente, $M=7,22, D P=2,89 \%, M=3,49$, $\mathrm{DP}=1,61 \%$, e $\mathrm{M}=7,39, \mathrm{DP}=3,02 \%$. O consumo de fibra dietética foi de $M=16,81, D P=10,57 \mathrm{~g} / \mathrm{dia}$ e de colesterol, $M=175,75, \mathrm{DP}=107,38 \mathrm{mg} / \mathrm{dia}$.

Em relação aos fatores de risco não tradicionais para DCV, identificou-se que os pacientes apresentavam níveis de PTHi expressos em mediana de 170pg/mL (9-2390), níveis médios de cálcio e fósforo de $M=9,78, D P=1,06 \mathrm{mg} / \mathrm{dL}$ e $\mathrm{M}=4,44, \mathrm{DP}=1,18 \mathrm{mg} / \mathrm{dL}$, respectivamente. O produto cálcio e fósforo expresso em mediana foi de $38,71 \mathrm{mg}^{2} / \mathrm{dL}^{2}(16,15-78,84)$ e o valor médio de hemoglobina foi de $M=11,35, D P=1,84 \mathrm{mg} / \mathrm{dL}$. 
$340 \mid$ M.H.L. GUSMÃO et al.

Tabela 1. Fatores de risco tradicionais para doença cardiovascular - demográficos, comportamentais e clínicos, em pacientes sob diálise peritoneal contínua com e sem sinais de desnutrição e inflamação. Salvador (BA), 2007.

\begin{tabular}{|c|c|c|c|c|c|c|c|c|c|}
\hline \multirow{3}{*}{ Variáveis } & \multicolumn{4}{|c|}{ Desnutridos } & \multicolumn{4}{|c|}{ Bom estado nutricional } & \multirow{3}{*}{$p$ valor } \\
\hline & \multicolumn{2}{|c|}{$\begin{array}{l}\text { Com Inflamação } \\
\qquad(n=13)\end{array}$} & \multicolumn{2}{|c|}{$\begin{array}{c}\text { Sem Inflamação } \\
(n=9)\end{array}$} & \multicolumn{2}{|c|}{$\begin{array}{l}\text { Com Inflamação } \\
(n=17)\end{array}$} & \multicolumn{2}{|c|}{$\begin{array}{c}\text { Sem Inflamação } \\
(n=22)\end{array}$} & \\
\hline & $n$ & $\%$ & $\mathrm{n}$ & $\%$ & $\mathrm{n}$ & $\%$ & $\mathrm{n}$ & $\%$ & \\
\hline \multicolumn{10}{|l|}{ Demográficos } \\
\hline Idosos & 4 & 30,8 & 4 & 44,4 & 8 & 47,1 & 6 & 27,3 & $0,56^{* * *}$ \\
\hline Gênero masculino & 6 & 46,2 & 5 & 55,6 & 8 & 47,1 & 6 & 27,3 & $0,40^{* * * *}$ \\
\hline \multicolumn{10}{|l|}{ Comportamentais } \\
\hline Etilismo & 4 & 30,8 & - & - & 4 & 23,5 & 2 & 9,1 & $0,16^{* * *}$ \\
\hline Ex-etilistas & 4 & 30,8 & 7 & 77,8 & 7 & 41,2 & 9 & 40,9 & $0,16^{* * *}$ \\
\hline Consumo elevado de álcool* & 2 & 50,0 & - & - & 2 & 50,0 & 2 & 100,0 & $0,43^{* * *}$ \\
\hline Tabagismo & 1 & 7,7 & 1 & 11,1 & - & - & - & - & $0,27^{* * *}$ \\
\hline Ex-tabagista & 5 & 38,5 & 1 & 11,1 & 7 & 41,2 & 8 & 36,4 & $0,45^{* * *}$ \\
\hline Consumo $\geq 20$ cigarros/dia** & 1 & 100,0 & - & - & - & - & - & - & $1,00^{* * *}$ \\
\hline Sedentarismo & 11 & 84,6 & 7 & 77,8 & 15 & 88,2 & 2 & 9,9 & $0,78^{* * *}$ \\
\hline \multicolumn{10}{|l|}{ Clínicos } \\
\hline Hipertensão & 10 & 76,9 & 5 & 55,6 & 15 & 88,2 & 17 & 77,3 & $0,31^{* * * *}$ \\
\hline Diabetes & 2 & 15,4 & 3 & 33,3 & 5 & 29,4 & 5 & 22,7 & $0,75^{* * *}$ \\
\hline Dislipidemia & 11 & 84,6 & 8 & 88,9 & 16 & 94,1 & 21 & 35,5 & $0,68^{* * * *}$ \\
\hline $\mathrm{CT} \geq 200 \mathrm{mg} / \mathrm{dL}$ & 6 & 46,1 & 4 & 44,4 & 9 & 52,9 & 12 & 54,5 & $0,93^{* * *}$ \\
\hline $\mathrm{TG} \geq 150 \mathrm{mg} / \mathrm{dL}$ & 6 & 46,1 & 5 & 55,5 & 11 & 64,7 & 16 & 72,7 & $0,44^{* * * *}$ \\
\hline \multicolumn{9}{|l|}{ e $<50 \mathrm{mg} / \mathrm{dL}$ (Mulheres) } & $0,56^{* * *}$ \\
\hline $\mathrm{LDL}-\mathrm{c} \geq 160 \mathrm{mg} / \mathrm{dL}$ & 3 & 23,1 & 1 & 11,1 & 8 & 47,0 & 7 & 31,8 & $0,25^{* * *}$ \\
\hline
\end{tabular}

*Considerados indivíduos etilistas; ${ }^{* *}$ Considerados indivíduos tabagistas; ${ }^{* * *}$ Teste qui-quadrado; ${ }^{* * * *}$ Teste exato de Fisher.

CT: colesterol total, TG: triglicérides, HDLc: fração de colesterol de lipoproteína de alta densidade, LDLc: fração de colesterol de lipoproteína de baixa densidade;

Nota: Sinal convencional utilizado; dado numérico igual a zero, não resultante de arredondamento.

Tabela 2. Fatores de risco tradicionais para doença cardiovascular - alimentares (M e DP) em pacientes sob diálise peritoneal contínua, com e sem sinais de desnutrição e inflamação. Salvador (BA), 2007.

\begin{tabular}{|c|c|c|c|c|c|c|c|c|c|}
\hline \multirow{3}{*}{ Variáveis } & \multicolumn{4}{|c|}{ Desnutridos } & \multicolumn{4}{|c|}{ Bom estado nutricional } & \multirow{3}{*}{$p$ valor } \\
\hline & \multicolumn{2}{|c|}{$\begin{array}{l}\text { Com Inflamação } \\
\qquad(n=13)\end{array}$} & \multicolumn{2}{|c|}{$\begin{array}{l}\text { Sem Inflamação } \\
\qquad(n=9)\end{array}$} & \multicolumn{2}{|c|}{$\begin{array}{l}\text { Com Inflamação } \\
\qquad(n=17)\end{array}$} & \multicolumn{2}{|c|}{$\begin{array}{c}\text { Sem Inflamação } \\
\qquad(n=22)\end{array}$} & \\
\hline & $\mathrm{M}$ & DP & $\mathrm{M}$ & DP & $\mathrm{M}$ & DP & $\mathrm{M}$ & DP & \\
\hline \multicolumn{10}{|l|}{ Fatores alimentares } \\
\hline VCT (kcal/dia) & 1489,91 & 273,62 & 1866,44 & 562,96 & 1881,67 & 661,96 & 1810,15 & 613,80 & $0,25^{* *}$ \\
\hline Carboidrato alimentar (\%VCT) & 43,33 & 11,01 & 49,38 & 9,58 & 48,69 & 9,48 & 47,51 & 7,71 & $0,36^{* *}$ \\
\hline Glicose absorvida do dialisato (\%VCT) & 20,14 & 8,49 & 14,67 & 8,58 & 17,95 & 10,07 & 14,87 & 8,40 & $0,31^{* *}$ \\
\hline Carboidratos totais (\%VCT & 63,48 & 8,50 & 64,27 & 8,90 & 66,64 & 6,61 & 62,39 & 9,07 & $0,46^{* *}$ \\
\hline Lipídeos (\%VCT) & 23,56 & 6,98 & 21,69 & 6,76 & 20,68 & 6,08 & 23,58 & 7,53 & $0,54^{* *}$ \\
\hline Gordura saturada (\%VCT) & 7,80 & 3,31 & 5,98 & 3,45 & 6,69 & 2,21 & 7,80 & 3,08 & $0,34^{* *}$ \\
\hline Gordura poli-insaturada (\%VCT) & 3,51 & 1,58 & 3,74 & 1,18 & 3,80 & 2,31 & 3,14 & 1,10 & $0,61^{* *}$ \\
\hline Gordura monoinsaturada (\%VCT) & 8,18 & 3,28 & 6,56 & 3,40 & 7,00 & 2,56 & 7,58 & 3,12 & $0,59^{* *}$ \\
\hline Fibras (g/dia) & 10,70 & 6,67 & 18,14 & 11,73 & 18,84 & 11,68 & 18,32 & 10,39 & $0,13^{* *}$ \\
\hline Colesterol (mg/dia) & 135,04 & 56,46 & 203,34 & 178,26 & 178,15 & 98,30 & 186,65 & 100,85 & $0,45^{* *}$ \\
\hline
\end{tabular}

** análise de variância ANOVA seguida do teste Tukey. VCT: valor calórico total; M: média; DP: desvio-padrão. 
Tabela 3. Fatores de risco não tradicionais para DCV, expresso em média e desvio-padrão ou em mediana e valor mínimo e máximo, pacientes sob contínua com e sem sinais de desnutrição e inflamação. Salvador (BA), 2007.

\begin{tabular}{|c|c|c|c|c|c|c|c|c|c|}
\hline \multirow{4}{*}{ Variáveis } & \multicolumn{4}{|c|}{ Desnutridos } & \multicolumn{4}{|c|}{ Bom estado nutricional } & \multirow{4}{*}{$p$ valor } \\
\hline & \multicolumn{2}{|c|}{$\begin{array}{c}\text { Com Inflamação } \\
(n=13)\end{array}$} & \multicolumn{2}{|c|}{$\begin{array}{c}\text { Sem Inflamação } \\
(\mathrm{n}=9)\end{array}$} & \multicolumn{2}{|c|}{$\begin{array}{c}\text { Com Inflamação } \\
(\mathrm{n}=17)\end{array}$} & \multicolumn{2}{|c|}{$\begin{array}{c}\text { Sem Inflamação } \\
\quad(n=22)\end{array}$} & \\
\hline & $\mathrm{M} /$ & $\mathrm{DP} /$ & $\mathrm{M} /$ & $\mathrm{DP} /$ & $\mathrm{M} /$ & DP/ & $\mathrm{M} /$ & $\mathrm{DP} /$ & \\
\hline & Mediana & VMM & Mediana & VMM & Mediana & VMM & Mediana & VMM & \\
\hline \multicolumn{10}{|l|}{$\begin{array}{l}\text { Metabolismo } \\
\text { cálcio e fósforo }\end{array}$} \\
\hline PTH $(p g / m L)^{\star \star}$ & 229,00 & $31-441$ & 170,00 & $25-263$ & 161,00 & $16,50-2390$ & 149,00 & $9,00-740$ & $0,54^{* * *}$ \\
\hline $\begin{array}{l}\text { Produto cálcio } \\
\text { e fósforo } \\
\left.\mathrm{mg}^{2} / \mathrm{dL}^{2}\right)^{\star \star}\end{array}$ & 42,72 & $16,15-75,33$ & 35,67 & $27,30-73,53$ & 39,84 & $24,65-78,84$ & 37,11 & $25,23-63,80$ & $0,63^{* * *}$ \\
\hline $\begin{array}{l}\text { Cálcio } \\
(\mathrm{mg} / \mathrm{dL})^{*}\end{array}$ & 10,00 & 0,76 & 9,90 & 1,26 & 9,72 & 0,94 & 9,65 & 1,24 & $0,80^{* * * *}$ \\
\hline $\begin{array}{l}\text { Fósforo } \\
(\mathrm{mg} / \mathrm{dL})^{*}\end{array}$ & 4,66 & 1,68 & 4,21 & 0,81 & 4,61 & 1,17 & 4,32 & 0,99 & $0,65^{* \star * *}$ \\
\hline $\begin{array}{l}\text { Anemia } \\
\text { Hemoblobina } \\
(\mathrm{mg} / \mathrm{dL})^{*}\end{array}$ & 10,93 & 1,86 & 12,26 & 2,05 & 11,15 & 1,47 & 11,37 & 2,00 & $0,39^{* * * *}$ \\
\hline
\end{tabular}

PTH: paratormônio; * Variável expressa em M e DP; ${ }^{* *}$ Variável expressa em Mediana e VMM; ${ }^{* * *}$ análise de variância ANOVA seguida do teste Tukey; ${ }^{* * * *}$ teste de Kruskal-Wallis seguido de Mann-Whitney.

DCV: doença cardiovascular; M: média; DP: desvio-padrão.

Quando se dividiu a população em grupos de acordo com a presença ou ausência de desnutrição e inflamação para avaliar a distribuição dos outros fatores de risco para DCV tradicionais - demográficos, comportamentais e clínicos (Tabela 1), alimentares (Tabela 2) e não tradicionais (Tabela 3), não foram evidenciadas diferenças entre os grupos estudados.

\section{I S C U S S Ã O}

Prevenir e tratar complicações cardiovasculares em pacientes em diálise têm sido considerado prioridade. Estratégias de prevenção e tratamento de complicações cardiovasculares nessa população necessitam de conhecimento dos fatores de risco e da relação entre eles ${ }^{6}$.

Ao iniciar o tratamento dialítico, o estado nutricional dos pacientes é preditor da sua evolução clínica' e constitui um fator de risco para DCV². A hipoalbuminemia determina alterações fisiopatológicas no metabolismo lipídico por meio da diminuição da pressão oncótica, o que estimula síntese hepática de albumina e outras proteínas, inclusive as apoliproteínas, determinando também alterações nas frações lipídicas².

Este estudo identificou que o deficit nutricional na população estudada ainda é frequente. Contudo os percentuais encontrados foram menores do que os registrados em alguns estudos, nos quais a desnutrição na forma leve a moderada se situa entre $39,2 \%$ e $51,2 \%$ e na forma grave entre $2,6 \%$ a $4,2 \% \%^{1,10-12}$. O nível sérico de albumina neste estudo também se encontrou abaixo do valor recomendado. Wang et al. ${ }^{12}$ também evidenciaram esse achado, contudo com níveis de albumina sérica menor do que na população avaliada. Diferenças da população, como características clínicas e menor tempo de diálise da população, podem ter contribuído para discrepância entre os resultados.

A desnutrição em pacientes em DP tem múltiplas causas e, dentre elas, registra-se a inflamação. Nessa situação, as proteínas de fase aguda, como a proteína C reativa e as citocinas pró-inflamatórias, exercem marcada influência sobre 
o estado nutricional, pois se associam à anorexia, aumento do catabolismo proteico e diminuição do anabolismo ${ }^{3}$.

Pacientes desnutridos em diálise frequentemente possuem sinais de inflamação, caracterizada por aumento nos níveis plasmáticos de Proteína C Reativa (PCR), que é um forte preditor de mortalidade por $\mathrm{DCV}^{3}$.

Neste estudo, foi observado que 49,2\% dos pacientes apresentavam níveis de PCR US considerados elevados $\left(>3 \mathrm{mg} / \mathrm{L}^{17}\right)$. A mediana do nível sérico de PCR US foi 2,5mg/L $(0,08-28,40)$, resultado que se aproxima do encontrado por Wang et $a{ }^{12}{ }^{12}$, em que a mediana de PCR US foi de $2,72 \mathrm{mg} / \mathrm{L}^{12}$.

Observou-se também no trabalho realizado que $21,3 \%$ dos pacientes estavam desnutridos e com processo inflamatório. Entre os desnutridos, os níveis de PCRUS eram maiores que aqueles do grupo com bom estado nutricional, resultado semeIhante ao encontrado por Stenvinkel et al. ${ }^{26}$. Esses pesquisadores, utilizando a ASG para classificar o estado nutricional de pacientes com DRC, observaram que o grupo desnutrido também apresentava níveis maiores de PCR US²6.

Merece atenção a elevada ocorrência de fatores de risco para DCV observada na população estudada. Essa tendência se manteve mesmo quando a amostra foi dividida em grupos de acordo com a presença ou ausência de desnutrição e inflamação. Apesar disso, não foi encontrada diferença entre os grupos estudados em relação à distribuição desses fatores de risco para DCV. Ressalta-se que o tamanho da amostra e alta prevalência dos fatores de rico nessa população podem ter contribuído para tal achado.

Dentre os fatores de risco comportamentais, observou-se prevalência de tabagismo de $3,3 \%$, enquanto outros estudos encontraram prevalência de 25\% a 37,0\% 5,12. Entretanto, a frequência de pacientes ex-tabagistas, ex-etilistas e sedentários foi elevada na população e nos grupos estudados. A temporalidade do estudo transversal deve ser considerada na interpretação dos resultados.
Em relação aos fatores de risco clínicos, a frequência de hipertensão foi elevada na população e em todos os grupos estudados. Prevalências de hipertensão de magnitude semelhante também foram descritas por outros estudos ${ }^{5,6}$. Assim, Gowdak et al. ${ }^{5}$, estudando fatores de risco cardiovascular em candidatos a transplante renal, encontraram um percentual de $74,0 \%$ de hipertensão arterial. Da mesma maneira, Longenecker et al. ${ }^{6}$ encontraram que $95,0 \%$ dos pacientes sob diálise eram portadores de hipertensão. A frequência de diabetes na população estudada foi de $24,6 \%$, enquanto Wang et al. ${ }^{12}$ evidenciaram frequência de 31,0\% em pacientes em DP.

Dislipidemia também foi evidenciada em altas proporções no presente estudo, assim como as alterações nas frações lipídicas. Resultados semeIhantes foram encontrados por Klafke et al. ${ }^{2}$. Esses pesquisadores observaram que, dos pacientes estudados, 53,0\% possuíam TG elevado, 68,0\% elevação do CT, 41,2\% elevação do LDL-c e 11,0\% redução do $\mathrm{HDL}-\mathrm{c}^{2}$. Ressalta-se que o valor de HDL-c encontrado por eles foi inferior ao do presente trabalho. Essa diferença pode estar relacionada a questões metodológicas, como a consideração de um ponto de corte único independente do gênero do paciente.

São escassos os dados na literatura sobre o consumo alimentar de pacientes em doença peritoneal. Inúmeros estudos epidemiológicos realizados nas últimas décadas têm demonstrado a estreita relação entre a casualidade de doenças crônicas não transmissíveis e fatores dietéticos ${ }^{27}$. No presente estudo, elevada contribuição energética dos carboidratos e alto consumo de gordura saturada, além do baixo consumo de gordura monoinsaturadas e de fibras da dieta, foram observados na população e nos grupos ${ }^{14}$. O elevado consumo de carboidrato alimentar - acima de $35 \%$ do $\mathrm{VCT}^{23}$ - contribuiu para a alteração do percentual desse macronutriente no VCT, já que a quantidade de glicose absorvida do dialisato se encontrou dentro dos valores estimados para o programa de diálise ${ }^{22,23}$. 
Entre os fatores de risco não tradicionais, sabe-se que a hiperfosfatemia, hipercalemia, elevação do produto cálcio e fósforo e hiperparatireoidismo constituem fatores de risco para calcificação vascular, isquemia e adversas complicações cardíacas $^{28}$. No presente estudo, os níveis médios de cálcio sérico estavam acima do recomendado pelo National Kidney Fondation ${ }^{24}$ em todos os grupos e na população. Resultados semelhantes foram observados por Noordzij et al. ${ }^{28}$.

Ademais, a média do nível sérico de hemoglobina apresentada pelos pacientes estava abaixo do recomendado somente no grupo desnutrido e inflamado ${ }^{25}$. É relevante considerar que a anemia constitui um fator importante na patogênese da hipertrofia ventricular, imprimindo ominoso prognóstico. A anemia é um achado comum nos pacientes renais crônicos devido à deficiência de eritropoetina e se associa de forma independente com o processo inflamatório nessa população ${ }^{29}$.

A associação entre o processo inflamatório e a desnutrição nos pacientes em diálise merece atenção, uma vez que ambas as condições podem contribuir para mortalidade por doença cardiovascular. Observa-se também que muitos dos fatores de risco evidenciados na população estudada, apesar de não apresentarem diferença quando analisados em grupos, de acordo com a presença ou ausência de desnutrição e inflamação, são passíveis de terapêutica, principalmente os relacionados com hábitos de vida.

\section{O N CLUS Ã O}

Os dados observados neste estudo mostram que a desnutrição e a inflamação são situações frequentemente observadas em pacientes sob DP. Entre os desnutridos, os níveis de PCRUS eram maiores que aqueles do grupo com bom estado nutricional. A elevada ocorrência de fatores risco para DCV observada na população estudada se manteve mesmo quando a amostra foi dividida em grupos de acordo com a presença ou ausência de desnutrição e inflamação. Contudo, nas condi- ções em que foi realizado o presente estudo, não foi encontrada diferença entre os grupos estudados em relação à distribuição desses fatores de risco para DCV.

Estudos com número maior de indivíduos e que busquem identificar a associação entre desnutrição na presença de inflamação e de outros fatores de risco para DCV se fazem necessários, já que o percentual de ocorrência desses fatores é alto nessa população, assim como trabalhos que contemplem entre suas variáveis as possíveis causas desses fatores de risco nessa população.

\section{A GRADECIMENTOS}

À equipe da unidade de diálise e laboratório do Complexo Hospitalar Universitário Professor Edgard Santos e da Clínica São Marcos. Ao Prof. Dr. Antonio Raimundo Pinto de Almeida. Ao Dr. Stênio Ataíde e à estatística Sandra Pinheiro.

\section{COLABORADORES}

M.H.L. GUSMÃO participou como mentora do trabalho científico, responsável pelo planejamento, execução e supervisão do projeto de pesquisa bem como pela elaboração do artigo. A.F.A. ALMEIDA, L.S.I. MICHELLE, M.N. MOREIRA e S.R. CAMPOS participaram da coleta e análise dos dados e da discussão dos resultados. L.J.C. PEREIRA participou da concepção do projeto de pesquisa e da discussão dos resultados. J.M.B. MEDEIROS responsável, como orientadora, pelo planejamento e supervisão do projeto de pesquisa.

\section{REFERÊ NCIAS}

1. CANADA-USA: peritoneal dialysis study group. Adequacy of dialysis and nutrition in continuous peritoneal dialysis: association with clinical outcomes. J Am Soc Nephrol. 1996; 7:198-207.

2. Klafke A, Moriguchi E, Barros EJ. Perfil lipídico de pacientes com insuficiência renal crônica em tratamento conservador, hemodiálise ou diálise peritoneal. J Bras Nefrol. 2005; 27(3):116-23.

3. Stenvinkel P. Inflammation in end-stage renal failure: could it be trated? Nephrol Dial Transplant. 
2002; 17(Suppl 8):33-8. doi:10.1093/ndt/17. suppl_8.33.

4. Canziani MEF. Doenças cardiovasculares na doença renal crônica. J Bras Nefrol. 2004; 26(3):20-1.

5. Gowdak LHW, Paula FJ, Giorgi DMA, Vieira MLC, Krieger EM, Lima JJG. Doença cardiovascular e fatores de risco cardiovascular em candidatos a transplante renal. Arq Bras Cardiol. 2005; 84(2): 156-60. doi: 10.1590/S0066-782X2005000200 012.

6. Longenecker JC, Coresh J, Powe NR, Levey AS, Fink NE, Martin A, et al. Traditional cardiovascular disease risk factors in dialysis patients compared with the general population: the choice study. J Am Soc Nephrol. 2002; 13:1918-27. doi: 10.1097/ 01.ASN.0000019641.41496.1E.

7. Pecoits-Filho R, Stevinkel P, Lindholm B, Bergstrom J, Noronha I, Abensur H. Desnutrição, inflamação e aterosclerose (síndrome MIA) em pacientes portadores de insuficiência renal crônica. J Bras Nefrol. 2002; 24(3):136-46.

8. Pecoits-Filho R, Lindholm B, Stenvinkel P. The malnutrition, inflammation, and atherosclerosis (MIA) syndrome: the heart of the matter. Nephrol Dial Transplant. 2002; 17(Suppl 11):28-31. doi: 10.1093/ndt/17.suppl_11.28.

9. Papagianni A, Kokolina E, Kalovoulos M, Vainas A, Dimitriadis C, Memmos D. Carotid atherosclerosis is associated with inflammation, malnutrition and intercellular adhesion molecule-1 in patients on continuous ambulatory peritoneal dialsis. Nephrol Dial Transplant. 2004; 19:1258-63. doi:10.1093/ ndt/gfh078.

10. Chan JYW, Che KI, Lam KMC, Chow K, Chung K, Li PK, et al. Comprehensive malnutrition inflammation score as a marker of nutritional status in Chinese peritoneal dialysis patientes. Nephology. 2007; 12(2):130-4. doi: 10.1111/j.1440-1797.20 06.00693.x.

11. Ghun HS, Lindholm B, Lee HB. Is malnutrition an independent predictor of mortality in peritoneal dialysis patients? Nephrol Dial Transplant. 2003; 18:2134-40. doi:10.1093/ndt/gfg318.

12. Wang AY, Woo J, Lam CW, Wang M, Chan IH, Gao $P$, et al. Associations of serum fetuin-A with malnutrition, inflammation, atherosclerosis and valvular calcification syndrome and outcome in peritoneal dialysis patients. Nephrol Dial Transplant. 2005; 20:1676-85. doi:10.1093/ndt/ gfh891.

13. National Kidney Foundation. Clinical practice guidelines for nutrition in chronic renal failure.
Adult Guidelines. Am J Kidney Dis. 2000; 35(Suppl 2):s1-140. doi:10.1053/kd.2000.6671.

14. Sposito AC, Caramelli B, Fonseca FAH, Bertolami MC. IV Diretriz brasileiras sobre dislipidemia e prevenção da aterosclerose do departamento de aterosclerose da Sociedade Brasileira de Cardiologia. Arq Bras Cardiol. 2007; 88(Suppl 1):2-19. doi: 10.1590/S0066-782X2007000700001.

15. Friedewald WT, Levy RJ, Fredrickson DS. Estimation of the concentration of low-density lipoprotein cholesterol in plama, without use of the preparative ultracentrifuge. Clin Chem. 1972; 18(6):499-502.

16. Castanheira M, Olinto MTA, Gigante DP. Associação de variáveis sócio-demográficas e comportamentais com a gordura abdominal em adultos: estudo de base populacional no Sul da Brasil. Cad Saúde Pública. 2003; 19(Supp 1):s55-s65. doi: 10.1590/S0102-311X2003000700007.

17. Santos RD. III Diretriz brasileiras sobre dislipidemia e diretriz de prevenção da aterosclerose do departamento de aterosclerose da Sociedade Brasileira de Cardiologia. Disponível. Arq Bras Cardiol. 2001; 77(Suppl 3):1-48. doi: 10.1590/S0066-782X20 01001500001.

18. Matsudo SM, Matsudo VR, Araújo T, Andrade D, Andrade E, Oliveira L, et al. Nível de atividade física da população do Estado de São Paulo: análise de acordo com gênero, idade, nível socioeconômico, distribuição geográfica e de conhecimento. Rev Bras Ciên Mov. 2002; 10(4):41-50.

19. Bressan J. Sistema de Suporte à Avaliação Nutricional e Prescrição de Dietas. $4^{\text {th }}$ ed. Agromídia Software - DietPro; 2003.

20. Instituto Brasileiro de Geografia e Estatística. Tabela de composição de alimentos. $4^{a}$ ed. Rio de Janeiro: IBGE; 1996.

21. Philippi ST. Tabela de composição de alimentos: suporte para decisão nutricional. Brasília: ANVISA; 2001.

22. Grodstein GP, Blumenkratz MJ, Kopple J, Morgan JK, Coburn JW. Glucose absorption during continuous ambulatory peritoneal dialysis. Kidney Int. 1981; 19 (4):564-67. doi:10.1038/ki.1981.53.

23. Toigo G, Aparicio M, Oattman P, Cano N, Cianciaruso $B$, Engel $B$, et al. Expert working group report on nutrition in adult patients with renal insufficiency (Part 2 of 2). Clin Nutr. 2000; 19(4): 281-91. doi:10.1054/clnu.2000.0129.

24. National Kidney Foundation. Clinical practice guideline for bone metabolism and disease in chronic kidney disease. Am J Kidney Dis. 2003; 42(Suppl 3):S1-S202. doi:10.1053/S0272-6386 (03)00905-3. 
25. National Kidney Foundation. Clinical practice guideline and clinical practice recommendations for anemia in chronic kidney disease: 2007 Update of Hemoglobin Target. Am J Kidney Dis. 2007; 50(3):471-530. doi:10.1053/j.ajkd.2007.06.008.

26. Stenvinkel $P$, Heimburger $O$, Paultre F, Diczfalusy $U$, Wang $T$, Berlund $L$, et al. Strong association between malnutrition, inflammation and atherosclerosis in chronic renal failure. Kidney Int. 1999; 55:1899-1911. doi:10.1046/j.1523-175 5.1999.00422.x.

27. Neumann AILCP, Shirassu MM, Fisberg RM. Consumo de alimentos de risco e proteção para doenças cardiovasculares entre funcionários públicos. Rev Nutr. 2006; 19(1):19-28. doi: 10.15 90/S1415-52732006000100002.
28. Noordzij M, Korevaar JC, Bos WJ, Boeschoten EW, Dekker FW, Bossuyt PM, et al. Mineral metabolism and cardiovascular morbidity and mortality risk: peritoneal dialysis patients compared with haemodialysis patients. Nephrol Dial Transplant. 2006; 21:2513-20. doi:10.1093/ndt/gfl257.

29. Choncho M, Lippi G, Montagnana M, Muggeo $M$, Targher $G$. Association of inflammation with anaemia in patients with chronic kidney disease not requiring chronic dialysis. Nephrol Dial Transplant. 2008; 23(9):2879-83. doi:10.1093/nd t/gfn109.

Recebido em: 16/4/2008

Versão final reapresentada em: 7/10/2009 Aprovado em: 17/3/2010 